\title{
Maleimides in the Cretaceous/Tertiary boundary sediments at Kawaruppu, Hokkaido, Japan
}

\author{
Akira Shimoyama,* Masaki Kozono, Hajime Mita and Shinya Nomoto \\ Department of Chemistry, University of Tsukuba, Tsukuba 305-8571, Japan
}

(Received July 2, 2001; Accepted August 24, 2001)

\begin{abstract}
Maleimides were detected in organic solvent extracts and chromic acid oxidation products of the Cretaceous/Tertiary boundary sediments at Kawaruppu. Free (organic solvent extractable) maleimides consisted of 2,3-dimethylmaleimide and 2-methyl-3-ethylmaleimide, and bound (oxidatively extractable) maleimides of 2-methylmaleimide and its 3 - $n$-alkyl homologs up to $\mathrm{C}_{4}$, benzomaleimide (phthalimide) and its methyl homologs. The total concentrations of free and bound maleimides ranged from n.d. to 10 and from 4 to $49 \mathrm{nmol} \mathrm{g}^{-1}$, respectively. The most abundant compound was 2-methyl-3-ethylmaleimide both in free and bound forms in all the sediments, indicating that the maleimides detected were very likely degradation products of porphyrins and/or chlorophylls. The concentrations of both forms of alkylmaleimides were significantly smaller in the boundary claystone than the sediments above and below it. Their depth profiles agreed with those of pristane plus phytane and $n$-alkanes, indicating smaller inputs of chlorophylls into the claystone, which relates to the large bio-mass extinction at the end of Cretaceous. The Kawaruppu sequence afforded the first case in which 2-methylmaleimide was the second most abundant maleimide, indicating a mild thermal history of the sediments. The ratios of 2,3-dimethyl- to 2methyl-3-ethylmaleimide of both free and bound forms showed constant values above and below the boundary claystone, reflecting the uniform diagenesis experienced by the sediments. The ratios exhibited larger values within the claystone, which is possibly related to different precursor chlorophylls caused by the massive extinction of organisms.
\end{abstract}

\section{INTRODUCTION}

Since Treibs discovered porphyrins in geological samples in 1934 (Treibs, 1934), much attention has been paid to these biomarkers of chlorophylls. The sedimentary porphyrins are usually complicated mixtures with various substituents on pyrrole rings as a result of diagenetic modification. The substitution patterns of petroleum porphyrins had been conveniently determined after decomposition to maleimides by the chromic acid oxidation method leaving alkyl groups unchanged (Ellsworth, 1970). In the oxidation products previously reported, a homologous series of 2-methylmaleimides with $n$-alkyl and branched alkyl side chains at 3-position were detected, and the most abundant maleimide was 2-methyl-3- ethylmaleimide (compound numbered $\mathbf{8}$ in Appendix, hereafter numbers in bold letter correspond to those shown in Appendix) (Martin et al., 1980; Barwise and Whitehead, 1980). The predominance was considered to reflect the fact that a 3-methyl4-ethylpyrrole ring (7) is a common constituent in most chlorophylls. Furlong and Carpenter (1988) had first applied the chromic acid oxidation for sediment samples to obtain 2-methyl-3ethylmaleimide (8) as a major maleimide, providing a potential use of the oxidative extraction to kerogen-bound porphyrins. Meantime, Grice et al. (1996, 1997) had found a series of 2-methyl-3alkylmaleimides, in which 2-methyl-3ethylmaleimide (2) predominated in organic solvent extracts of Permian and Mid-Triassic marine sediments. These solvent-extractable maleimides

*Corresponding author (e-mail: ashimoya@staff.chem.tsukuba.ac.jp) 
evidently originated from porphyrins in sediments, but their formation process, which should involve an oxidation step, has not been elucidated.

Since maleimides can be important biomarker compounds derived from chlorophylls, it is of interest to disclose their kinds and concentrations over a sedimentary sequence, something which has rarely been done. We recently found maleimides of free (solvent extractable) and bound (oxidatively extractable) forms in the Neogene Shinjo sediments located at an old oil field and revealed the depth profiles of their concentrations over the sequence (Kozono et al., 2001). We then concluded that bound maleimides were derived from kerogen-bound porphyrins and better reflect the thermal history of the sediments compared to the free maleimides which are liable to migration, modification, and decomposition by sediment constituents and heat during burial.

The Cretaceous/Tertiary (K/T) boundary sediments have been interesting in connection with the massive extinction of organisms ranging from dinosaurs to marine plankton. The global extinction event has been postulated to be due to an impact by an extraterrestrial body (Alvarez et al., 1980; Smit and Hertogen, 1980) or to extensive volcanism (Officer and Drake, 1983; McLean, 1985). The K/T boundary sediments at Kawaruppu provide a good sedimentary sequence for organic geochemical studies. The boundary claystone is thicker (nearly $14 \mathrm{~cm}$ ) than those at other sites (e.g., Stevns Klint in Denmark, Gubbio in Italy and Woodside Creek in New Zealand) and can be analyzed for organic compounds in detail along its depth. In the present study we analyzed free and bound maleimides in the $\mathrm{K} / \mathrm{T}$ boundary sediments at Kawaruppu in order to obtain another example illustrating the utility of maleimides as biomarkers, as well as to examine maleimide distribution in a sedimentary sequence differing in maturity from that of the Shinjo sediments. Previous studies indicated that the 65 million year diagenesis has taken place evenly over the sediments throughout the sequence (Mita and Shimoyama, 1999a; Katsumata and Shimoyama, 2001). Organic geochemical studies of amino ac- ids (Mita et al., 1996) and dicarboxylic acids (Mita et al., 1998) in the sediments were discussed as to the possibility of the presence of extraterrestrial organic compounds as well as terrestrial ones. Characteristic distributions of aliphatic hydrocarbons (Mita and Shimoyama, 1999b) and polycyclic aromatic hydrocarbons (PAHs) (Mita and Shimoyama, 1999a) in the sediments were reported and related to the massive extinction and wildfires, respectively.

\section{EXPERIMENTAL}

\section{Samples}

The K/T boundary sediments occurring near Kawaruppu Town, Tokachi District, Hokkaido, and their location and lithology were reported by Saito et al. (1986), and Kaiho and Saito (1986). Briefly, the sedimentary sequence at Kawaruppu consists predominantly of a marine, dark gray siltstone, bearing occasional calcareous concretions. The boundary claystone is grayish black and nearly $14 \mathrm{~cm}$ thick. These sediment samples were kindly provided by Prof. Y. Kajiwara of the University of Tsukuba. A total of 22 sediment samples whose horizons were reported by Mita $e t$ al. $(1996,1998)$ were analyzed for this study. They included 11 samples within the boundary claystone $(0.8-13.7 \mathrm{~cm}$ in relative depth), 5 samples above $(40-495 \mathrm{~cm})$ and 6 samples below $(-395-0 \mathrm{~cm})$ the boundary claystone.

\section{Analyses}

For each analysis, $1.0 \mathrm{~g}$ of powdered sample was extracted by sonication with $3 \mathrm{ml}$ of a mixture of dichloromethane and acetone $(9: 1, \mathrm{v} / \mathrm{v})$. After centrifugation, the supernatant was saved. The extraction and centrifugation processes were repeated four times. The extracts were combined and carefully concentrated under reduced pressure and were applied to a silica gel column $(175 \mathrm{~mm}$ $\times 10 \mathrm{~mm}$ i.d.). Elution was carried out with hexane, dichloromethane and then, a mixture of dichloromethane and acetone $(9: 1, \mathrm{v} / \mathrm{v})$. Maleimides were recovered in the final eluent. The eluent was concentrated to $50 \mu$ under a stream 
of nitrogen, and analyzed by a gas chromatograph combined with a mass spectrometer (GC-MS). Maleimides recovered from this fraction were named free maleimides.

The sediment samples recovered after the above extraction were oxidized with chromic acid according to the method of Furlong and Carpenter (1988), as follows. To a suspension of the sediment sample in $10 \mathrm{ml}$ of $25 \%$ sulfuric acid (w/v) were added $10 \mathrm{ml}$ of $10 \%$ chromium trioxide (w/v) below $0^{\circ} \mathrm{C}$. The suspension was stirred for 2 hours below $0^{\circ} \mathrm{C}$ and then for 2 hours at room temperature. After centrifugation, the supernatant was recovered and extracted five times by shaking with $5 \mathrm{ml}$ of benzene. The benzene extracts were combined and concentrated carefully to 50 $\mu \mathrm{l}$ under nitrogen flow and analyzed by GC-MS. Maleimides obtained from the oxidative extraction of the solvent-extracted sediment samples were named bound maleimides.

Hexane and chromium trioxide used were of analytical grade (Wako pure chemical). Other organic solvents, such as dichloromethane, acetone and benzene, were doubly distilled before use. Water used as the solvent for chromic acid oxidation was obtained by successive distillation and deionization. To avoid laboratory contamination, all glassware was heated at $500^{\circ} \mathrm{C}$ for 3 hours before use.

The samples were analyzed by GC-MS using a Hewlett Packard G1800A GCD system. The GC was equipped with a FFAP bonded FS-WCOT capillary column $(60 \mathrm{~m} \times 0.25 \mathrm{~mm}$ i.d. $)$. The GC oven temperature was programmed to give $60^{\circ} \mathrm{C}$ for $2 \mathrm{~min}$, to increase from $60^{\circ} \mathrm{C}$ to $180^{\circ} \mathrm{C}$ at a

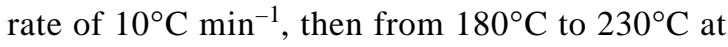
a rate of $3^{\circ} \mathrm{C} \mathrm{min}^{-1}$, and to hold at the final temperature for $50 \mathrm{~min}$. Helium was used as a carrier

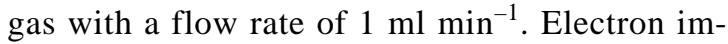
pact (at $70 \mathrm{eV}$ ) mass spectra were obtained by scanning from $\mathrm{m} / \mathrm{z} 45$ to 425 every $0.5 \mathrm{~s}$.

Identification of maleimides was made by comparison of GC retention times and of MS fragment patterns with those of standard compounds: 2methylmaleimide, 2,3-dimethylmaleimide, 2methyl-3-ethylmaleimide, benzomaleimide (phthalimide), $\alpha$-methylbenzomaleimide (3methylphthalimide), $\beta$-methylbenzomaleimide (4methylphthalimide). These compounds were prepared in our laboratory (Nomoto et al., 2001), except for benzomaleimide (Wako pure chemical). Concentrations of maleimides were estimated by comparing peak areas on mass fragmentograms of molecular ions to those of the standard compounds.

\section{RESULTS AND DISCUSSION}

\section{Identification and concentrations}

The total ion chromatogram and mass fragmentograms of the oxidative extract of the sample at the top horizon $(12.7-13.7 \mathrm{~cm})$ of the claystone are shown in Fig. 1. The mass fragmentograms show the presence of eight maleimides. These compounds include a series of 2-methylmaleimides with $n$-alkyl substituents up to $\mathrm{C}_{4}$ and benzomaleimides including $\alpha$ - and $\beta$ methylbenzomaleimides. 2-Methyl-3propylmaleimide and 2-methyl-3-butylmaleimide were identified by comparing their mass spectra with those reported by Barwise and Whitehead (1980), and Martin et al. (1980). The maleimides shown in the figure were found in the oxidative extracts from the sediments, and the direct extracts contained only 2,3-dimethylmaleimide (1) and 2methyl-3-ethylmaleimide (2). Maleimide with no substituent and those with branched alkyl side chains were not detected in the sediments.

Table 1 gives the concentrations of free and bound maleimides. Almost all the sediments contained maleimides in free or bound form ranging in concentration from n.d. to 10 and from 4 to 49 nmol $\mathrm{g}^{-1}$, respectively. The concentrations of free maleimides were about one order of magnitude smaller than those of the bound ones. The maximum concentrations of 2-methyl-3ethylmaleimide, the most abundant maleimide in both free and bound forms, were 4.6 and 23 nmol $\mathrm{g}^{-1}$, respectively. The concentrations of bound benzomaleimide (10) in the sediments above and below the boundary claystone were one or two orders of magnitude smaller than those of 


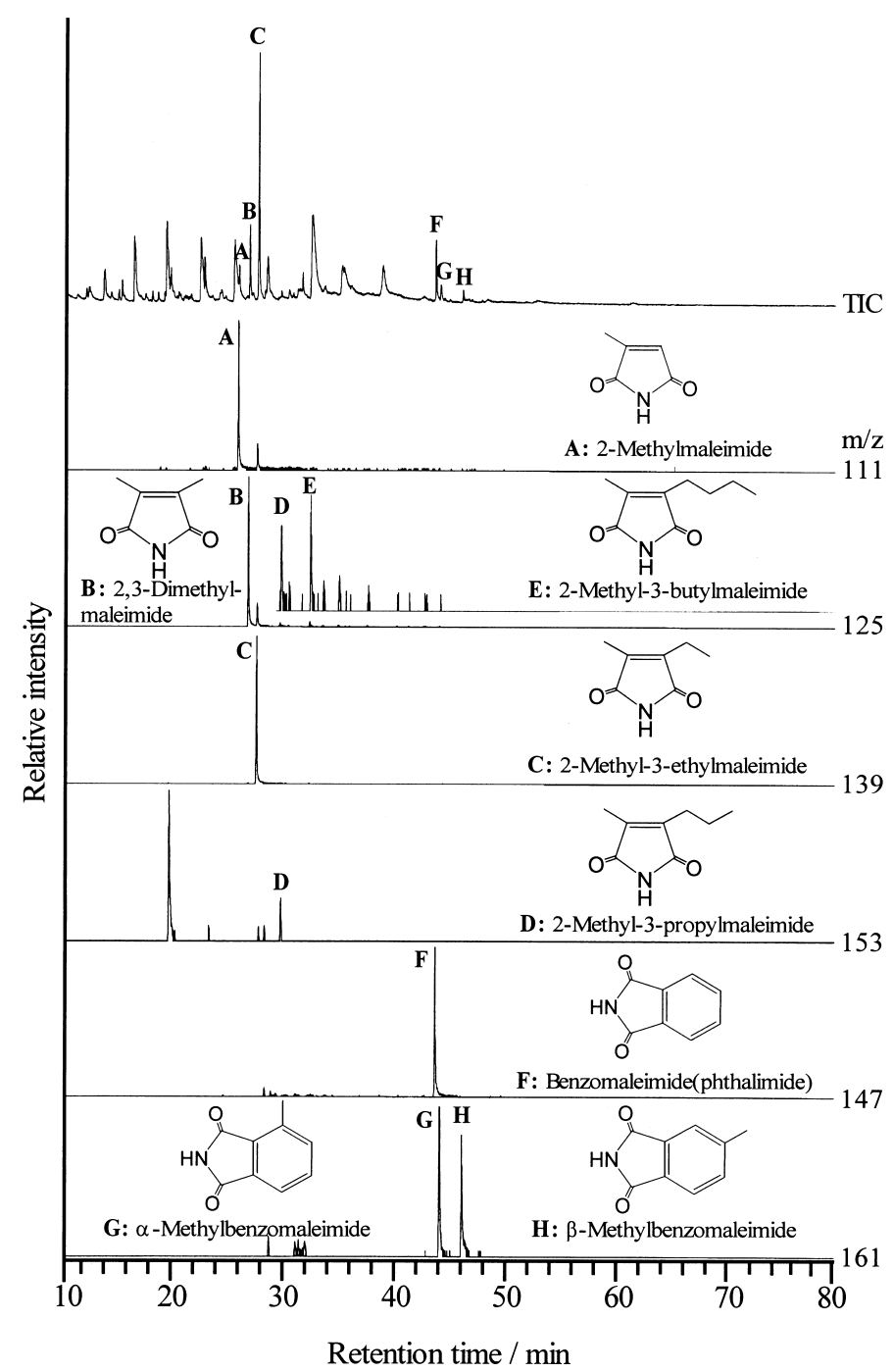

Fig. 1. Total ion chromatogram and mass fragmentograms of bound maleimides recovered from the sediment at the top horizon $(12.7-13.7 \mathrm{~cm})$ of the K/T boundary claystone at Kawaruppu, Japan.

2-methyl-3-ethylmaleimide (8). 2-Methyl-3propylmaleimide and 2-methyl-3-butylmaleimide, identified but not quantified, were even lower in concentration than methylbenzomaleimides (12) as judged from their peak areas on the mass fragmentograms.

\section{Characteristics of free maleimides}

Free maleimides consisted of 2,3dimethylmaleimide (1) and 2-methyl-3ethylmaleimide (2), and their concentrations in the
22 sediments over the stratigraphic sequence are plotted in Fig. 2, along with those of pristane plus phytane and $n$-alkanes (Mita and Shimoyama, 1999b). The concentrations of free maleimides decreased significantly at the lowest horizon in the boundary claystone from those in the sediments below the claystone, and were negligible in the claystone as seen in Fig. 2(a). Their concentrations then sharply increased around the top horizon of the claystone. The depth profile of the free maleimides is very similar to those of pristane 


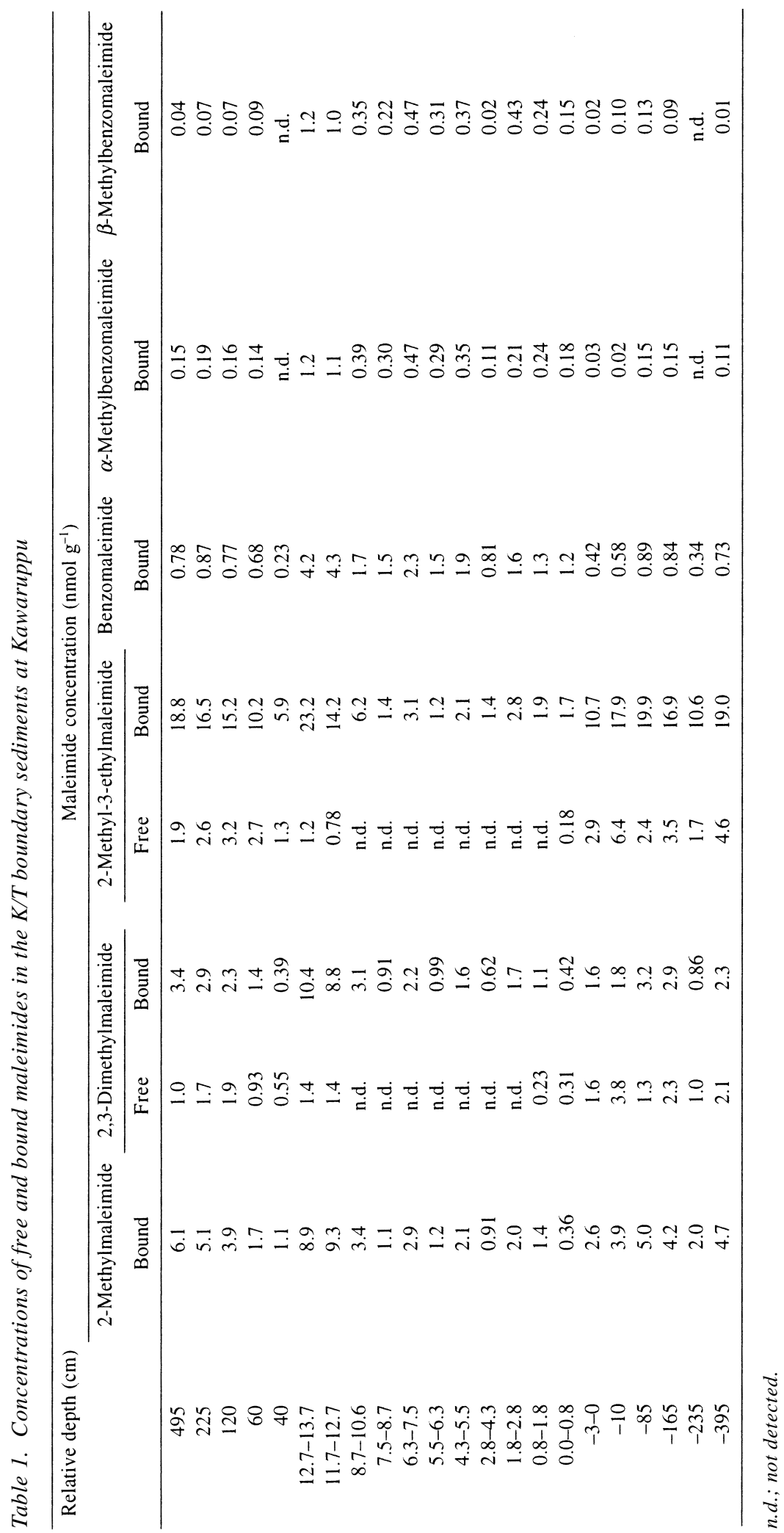




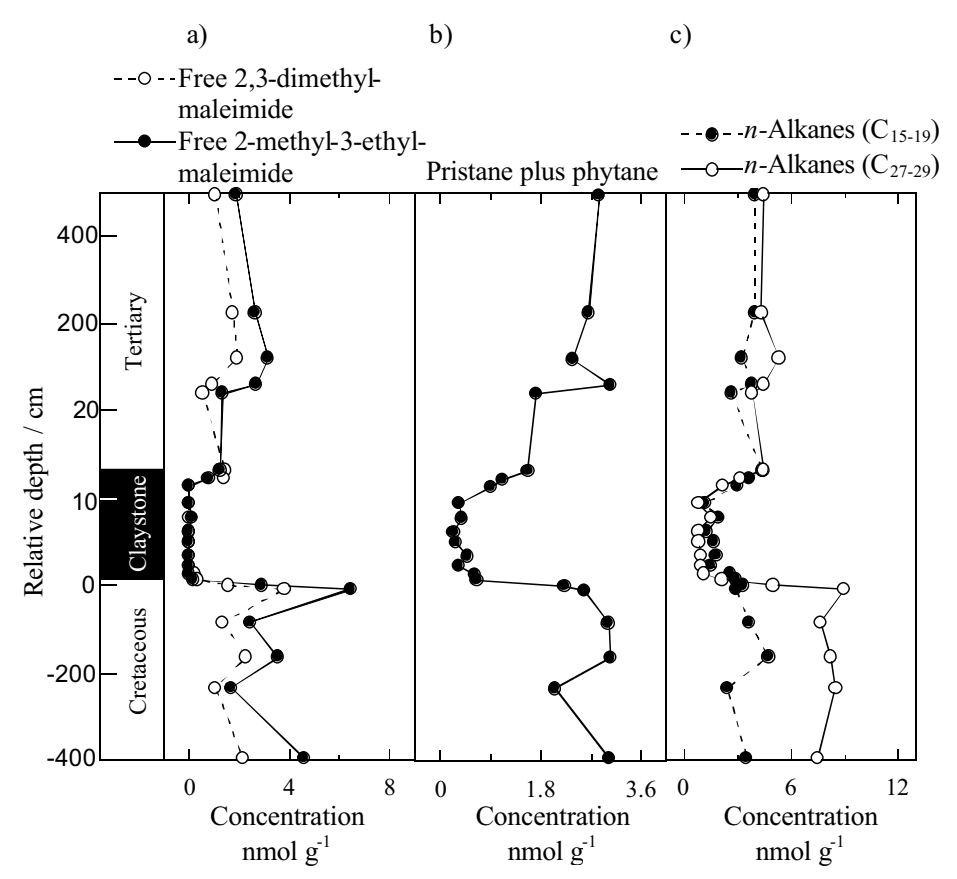

Fig. 2. Depth profiles of free maleimides $(a)$, pristane plus phytane $(b)$, and shorter chain n-alkanes $\left(C_{15}\right.$ to $\left.C_{19}\right)$ and longer ones $\left(C_{27}\right.$ to $\left.C_{29}\right)(c)$ in the K/T boundary sediments at Kawaruppu, Japan. The data depicted in (b) and (c) are from Mita and Shimoyama (1999b).

and phytane (Fig. 2(b)), which are mainly derived from chlorophylls. This fact and the predominance of 2-methyl-3-ethylmaleimide (2) indicates that the free maleimides found in the sediments are of chlorophyll origin.

In a separate study we examined maleimides in the Neogene Shinjo sediments, in which burial diagenesis is known to have greatly affected the organic components (Kozono et al., 2001). The free maleimides significantly decreased with depth, as is distinct from the increasing trend of $n$-alkanes and TOC. That finding suggested that the free maleimides are not stable during catagenesis that generated petroleum. In the Kawaruppu sedimentary sequence, where diagenesis is inferred to have taken place evenly over the whole sequence (Mita and Shimoyama, 1999a; Katsumata and Shimoyama, 2001), the depth profiles of free maleimides likely reflect the input of chlorophylls. The decrease in the concentration of free maleimides within the claystone can be related to a smaller input of their precursor chlo- rophylls into the claystone than into the sediments above and below it. In a previous study, both shorter chain $\left(\mathrm{C}_{15}\right.$ to $\left.\mathrm{C}_{19}\right)$ and longer chain $n$-alkane $\left(\mathrm{C}_{27}\right.$ to $\left.\mathrm{C}_{29}\right)$ were reported to show similar depth profiles (Fig. 2(c)), indicating smaller inputs of organic matter from marine algae and terrestrial plants, respectively, into the boundary claystone than into the sediments above and below it (Mita and Shimoyama, 1999b).

\section{Characteristics of bound maleimides}

The concentrations of bound maleimides are plotted in Fig. 3. The predominance of 2-methyl3-ethylmaleimide (8) suggests that these maleimides are derived from chlorophylls incorporated into kerogen, probably as porphyrins or their diagenetically modified forms (Barakat and Yen, 1989; Barakat and Rullkötter, 1998). If this is the case, the depth profile of the bound alkylmaleimides represents that of porphyrins. The concentrations of bound forms were larger than those of free maleimides in all the sediments, but 


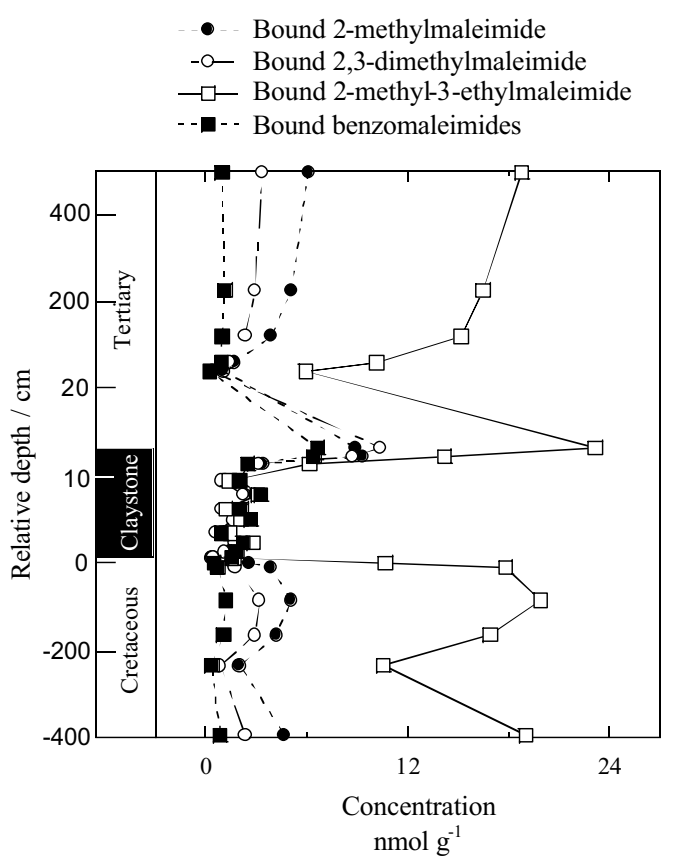

Fig. 3. Depth profiles of bound maleimides in the K/T boundary sediments at Kawaruppu, Japan.

smaller in the boundary claystone than in the sediments above and below. Although recoveries of maleimides in the oxidative extraction process are unknown, the good agreement of the depth profile of the bound alkylmaleimides with those of the free maleimides and pristane plus phytane indicates that the bound alkylmaleimides were obtained from all the sediments with constant efficiency of the oxidation and extraction process. Bound 2-methyl-3-ethylmaleimide (8), which is apparently of chlorophyll origin, may be better preserved in kerogen-bound porphyrins than as a maleimide molecule in the sediments. The depth distributions of the bound alkylmaleimides show a similar pattern to those of free ones as seen in Fig. 3, again reflecting the input of chlorophylls into the sediments.

Bound benzomaleimide (10) and its $\alpha$ - and $\beta$ methyl homologs (12) were detected in the sediments in smaller amounts than those of bound alkylmaleimides as seen in Fig. 3. Benzomaleimides, first found by Barwise and Whitehead (1980) as an oxidation product of pe- troleum porphyrins, provide chemical evidence for the presence of sedimentary benzoporphyrins, or so-called rhodoporphyrins (Kaur et al., 1986). Benzoporphyrins could not be correlated directly to chlorophylls, because no chlorophyll with a benzene ring (9) fused to a tetrapyrrole skeleton is known in organisms. We also found bound benzomaleimides (10) in the Shinjo sediments, where the ratio of benzomaleimide to alkylmaleimides increased with depth, showing benzomaleimide to be the most abundant maleimide in the intense oil generation zone (Kozono et al., 2001). The depth profiles of bound benzomaleimides (10) in the Kawaruppu sediments were different from those of bound alkylmaleimides, and exhibited a slight increase in the claystone (Table 1). The low concentration of bound benzomaleimides (10) throughout the sequence may indicate a mild thermal history of the sediments, since benzoporphyrins were revealed to be formed mainly by catagenesis in the case of the Shinjo sediments, as stated above.

It should be noted that bound 2methylmaleimide (4) was the second most abundant in this sedimentary sequence. There have been no previous reports describing bound 2methylmaleimide (4) as the second most abundant bound maleimide obtained from petroleum or sediments, in which bound 2,3-dimethylmaleimide (6) was usually detected as the second one. The concentration of bound 2-methylmaleimide (4) was lower than those of 2-methyl-3-ethyl- (8) and 2,3-dimethylmaleimide (6) in the Shinjo sediments (Kozono et al., 2001). 2,3-Dimethyl- (6) and 2methylmaleimide (4) do not have corresponding chlorophylls. In this study, we found in heating experiments of etioporphyrin that the transformation of a 3-methyl-4-ethyl- (7) into a 3,4dimethylpyrrole unit (5) proceeds smoothly above $250^{\circ} \mathrm{C}$ in the presence of clay catalysts without formation of measurable amounts of a 3methylpyrrole unit (3) (details of this will be reported elsewhere). Therefore, bound 2 methylmaleimide (4) could hardly be formed by demethylation of bound 2,3-dimethylmaleimide (6). However, a possibility of further 
demethylation of bound dimethylmaleimide during diagenesis of the $\mathrm{K} / \mathrm{T}$ sediments cannot be completely excluded.

On the other hand, 3-methylpyrrole-containing porphyrins, which afford bound 2methylmaleimide (4) on oxidation, have been found in petroleum and sediments (Ocampo et al., 1992). Several possibilities have been proposed for their formation pathways from chlorophyll $c$ (Ocampo et al., 1984; Verne-Mismer et al., 1988) and from the methylvinylpyrrole moiety of many chlorophylls (Sundararaman and Boreham, 1990). Since these mechanisms require the presence of rather labile functional groups, such as a vinyl, a keto, and a carboxyl group in the precursor chlorophylls or geoporphyrins, it is possible that early diagenesis generated 3-methylpyrrole-containing porphyrins, which have been preserved in kerogen in amount exceeding the production of a 3,4dimethylpyrrole unit (5) by subsequent diagenesis. This may imply that the sediments in this sequence have a milder diagenetic history than those containing bound 2,3-dimethylmaleimide (6) in excess of bound 2-methylmaleimide (4), since the formation of the former requires stronger diagenesis as compared to that of the latter.

\section{Comparison of free and bound 2-methylmaleimide distribution}

A clear account cannot be given, at present, for the absence of free 2-methylmaleimide in the Kawaruppu sediments, which showed considerable concentrations of its bound form. Grice $e t$ al. (1996) failed to detect free 2-methylmaleimide among a series of free 2-methyl-3alkylmaleimides discovered for the first time in Permian and Mid-Triassic sediments. As the second case of free maleimide detection, we found free 2-methylmaleimide in a limited number of samples of the Neogene Shinjo sediments in lower concentration than that of bound 2methylmaleimide (4) (Kozono et al., 2001). On the contrary, bound 2-methylmaleimide (4) had always been contained among the previously reported bound maleimides in appreciable concentrations (Martin et al., 1980; Barwise and
Whitehead, 1980; Kozono et al., 2001). Further studies should be performed to elucidate the transformation process of chlorophylls or porphyrins into free maleimides.

\section{Effect of diagenesis inferred from alkylmaleimide ratio}

The ratios of 2,3-dimethyl- to 2-methyl-3ethylmaleimide expressed as 2,3dimethylmaleimide/(2,3-dimethyl- + 2-methyl-3ethylmaleimide) with the depth of the sediments are plotted in Fig. 4, together with isomer ratios of methylnaphthalenes, methylphenanthrenes (Mita and Shimoyama, 1999a) and methyldibenzothiophene (Katsumata and Shimoyama, 2001). We have shown this ratio of bound maleimides to relate to the maturity of organic matter in the Shinjo sediments, and have called it "demethylation index of 2-methyl-3ethylmaleimide" (Kozono et al., 2001). The index is based on feasibility of allylic bond cleavage in the structure of 3-methyl-4-ethylpyrrole unit (7) by diagenesis. The demethylation indices (Fig. 4(a)) of both free and bound 2-methyl-3ethylmaleimide in the Kawaruppu sediments showed nearly constant values above and below the boundary claystone, reflecting the uniform diagenesis experienced by the sediments, and manifested also in the isomer ratios of polycyclic aromatic hydrocarbons (Fig. 4(b)) (Mita and Shimoyama, 1999a) and methyldibenzothiophene (Fig. 4(c)) (Katsumata and Shimoyama, 2001). The sensitivity of the index of the free maleimide over that of the bound is reproduced in this case without scattering.

On the other hand, the above indices exhibited larger values within the claystone, although all those of the free maleimides were not obtained due to their absence from many sediments. Although bound 2,3-dimethylmaleimide (6) has no corresponding chlorophylls, it can be generated from many bound maleimide structures including bound 2-methyl-3-ethylmaleimide (8) (Barakat and Yen, 1989; Verne-Mismer et al., 1988; Kozono et al., 2001). Therefore, it may be that differences in the precursors, in relation to the massive ex- 
a)

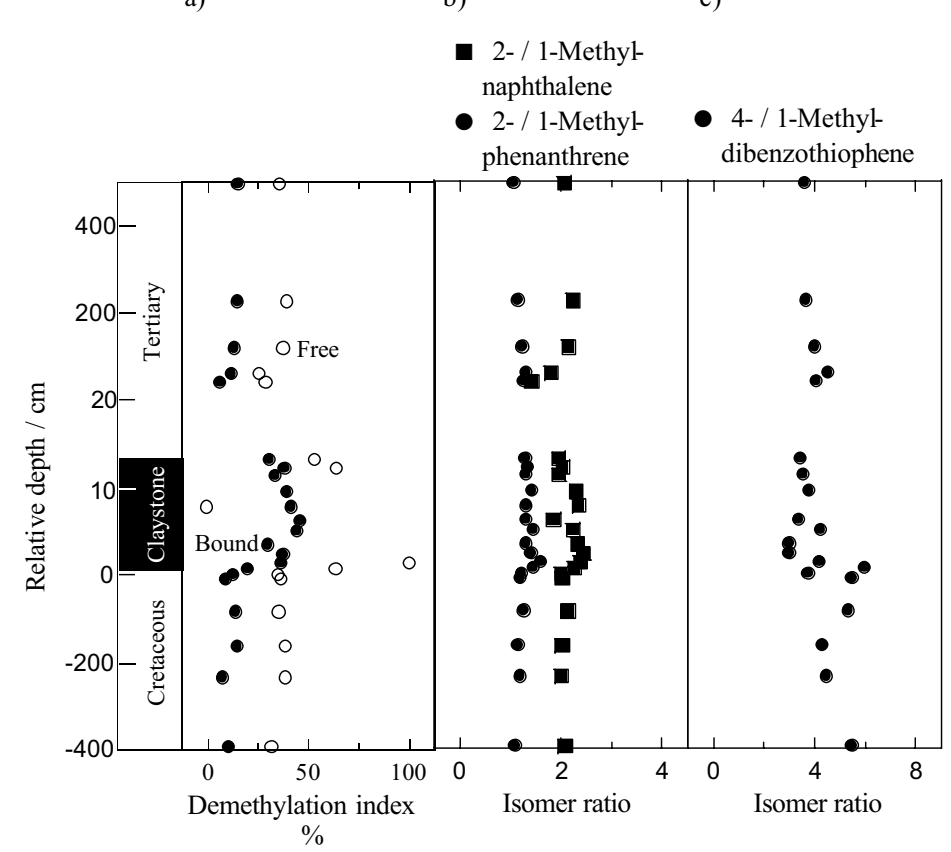

Fig. 4. Depth profiles of demethylation index (2,3-dimethylmaleimide/(2,3-dimethyl- + 2-methyl-3-ethylmaleimide)) (a), isomer ratios of 2- to 1-methylnaphthalene, methylphenanthrene (b), and 4- to 1-methyldibenzothiophene (c) in the K/T boundary sediments at Kawaruppu, Japan. The data depicted in (b) is from Mita and Shimoyama (1999a) and (c) from Katsumata and Shimoyama (2001).

tinction and the resulting change of biological distribution (Saito et al., 1986), affected the demethylation indices in the claystone in a sedimentary sequence that has a mild and uniform thermal history.

\section{CONCLUSIONS}

Maleimides were analyzed in 22 samples from the K/T boundary sediments at Kawaruppu. These included 11 samples within the boundary claystone, 5 samples above and 6 samples below the boundary claystone. Examination of their characteristics led the following conclusions

1. The sediments contained 2methylmaleimide (4) and its 3 - $n$-alkyl homologs up to $\mathrm{C}_{4}(\mathbf{6}, \mathbf{8})$, benzomaleimide $(\mathbf{1 0})$ and its methyl homologs (12) in bound forms. Their concentrations were from 4 to $49 \mathrm{nmol} \mathrm{g}^{-1}$. The concentrations of free maleimides, which consisted only of 2,3-dimethylmaleimide (1) and 2- methyl-3-ethylmaleimide (2), varied up to 10 nmol $\mathrm{g}^{-1}$. In most sediments the most predominant maleimide was 2-methyl-3-ethylmaleimide $(\mathbf{2}, \mathbf{8})$, which is likely derived from 3-methyl-4ethyl-pyrrole units (7) included in many chlorophylls and the major sedimentary porphyrins.

2. The concentrations of both forms of alkylmaleimides were significantly smaller in the boundary claystone than in the sediments above and below. The depth profiles of these alkylmaleimides agreed with those of pristane plus phytane and $n$-alkanes, indicating smaller inputs of chlorophylls into the claystone.

3. The depth profiles of bound benzomaleimide (10) and its $\alpha$ - and $\beta$-methyl homologs (12) were different from those of bound alkylmaleimides and exhibited a slight increase in the claystone. They were detected in the sediments in smaller amounts than those of bound alkylmaleimides, possibly due to a mild thermal history of the sediments throughout the sequence. 
4. Bound 2-methylmaleimide (4) was the second most abundant in this sedimentary sequence, as is distinct from the previous studies in which 2,3-dimethylmaleimide (6) was the second most abundant. Bound 2-methylmaleimide (4) was supposed to have been generated by early diagenesis and to have been preserved in kerogen in larger amounts than diagenetically produced 2,3dimethylmaleimide (6).

5. The ratios of 2,3-dimethyl- to 2-methyl-3ethylmaleimide of both free and bound forms showed constant values above and below the boundary claystone, reflecting the uniform diagenesis experienced by the sediments. The ratios exhibited larger values within the claystone than in the sediments above and below, which is possibly related to difference in the precursors.

Acknowledgments-We thank Prof. Y. Kajiwara, University of Tsukuba, for samples of the K/T boundary sediments at Kawaruppu. We are grateful to Prof. J. R. Cronin, Arizona State University, who read an earlier version of this paper and provided thoughtful suggestions in addition to improved English usage. This work was supported by the Grant-in-Aid for Scientific Research (B) No. 11440166 from the Ministry of Education, Science, Sports and Culture, Japan.

\section{REFERENCES}

Alvarez, L. W., Alvarez, W., Asaro, F. and Michel, H. V. (1980) Extraterrestrial cause for the CretaceousTertiary extinction. Science 208, 1095-1108.

Barakat, A. O. and Rullkötter, J. (1998) Product distribution from oxidative degradation of sulphur-rich kerogens from the Nordlinger Ries (southern Germany). Fuel 77, 85-94.

Barakat, A. O. and Yen, T. F. (1989) The nature of porphyrins in kerogen. Evidence of entrapped etioporphyrin species. Energy and Fuels 3, 613-616.

Barwise, A. J. G. and Whitehead, E. V. (1980) Separation and structure of petroporphyrins. Advances in Organic Geochemistry 1979 (Maxwell, J. R. and Douglas, A. G., eds.), 181-192, Pergamon Press, Oxford.

Ellsworth, R. K. (1970) Gas chromatographic determination of some maleimides produced by the oxidation of heme and chlorophyll a. J. Chromatog. 50, 131-134.

Furlong, E. T. and Carpenter, R. (1988) Pigment pres- ervation and remineralization in oxic coastal marine sediments. Geochim. Cosmochim. Acta 52, 87-99.

Grice, K., Gibbison, R., Atkinson, J. E., Schwark, L., Eckardt, C. B. and Maxwell, J. R. (1996) Maleimides (1H-pyrrole-2,5-diones) as molecular indicators of anoxygenic photosynthesis in ancient water columns. Geochim. Cosmochim. Acta 60, 3913-3924.

Grice, K., Schaeffer, P., Schwark, L. and Maxwell, J. R. (1997) Changes in palaeoenvironmental conditions during deposition of the Permian Kupferschiefer (Lower Rhine Basin, northwest Germany) inferred from molecular and isotopic compositions of biomarker components. Org. Geochem. 26, 677-690.

Kaiho, K. and Saito, T. (1986) Terminal Cretaceous sedimentary sequence recognized in the northernmost Japan based on planktonic foraminiferal evidence. Proc. Jpn. Acad. 62(B), 145-148.

Katsumata, H. and Shimoyama, A. (2001) Thiophenes in the Cretaceous/Tertiary boundary sediments at Kawaruppu, Hokkaido, Japan. Geochem. J. 35, 6776 .

Kaur, S., Chicarelli, I. and Maxwell, J. R. (1986) Naturally occurring benzoporphyrins: Bacterial marker pigments? J. Am. Chem. Soc. 58, 1325-1329.

Kozono, M., Nomoto, S., Mita, H. and Shimoyama, A. (2001) Detection of maleimides and their characteristics in Neogene sediments of the Shinjo basin, Japan. Geochem. J. 35, 225-236.

Martin, J., Quirke, E., Shaw, G. J., Soper, P. D. and Maxwell, J. R. (1980) Petroporphyrins-II. The presence of porphyrins with extended alkyl substituents. Tetrahedron 36, 3261-3267.

McLean, D. M. (1985) Deccan traps mantle degassing in the terminal Cretaceous marine extinctions. Cret. Res. 6, 235-259.

Mita, H. and Shimoyama, A. (1999a) Distribution of polycyclic aromatic hydrocarbons in the K/T boundary sediments at Kawaruppu, Hokkaido, Japan. Geochem. J. 33, 305-315.

Mita, H. and Shimoyama, A. (1999b) Characterization of $n$-alkanes, pristane and phytane in the Cretaceous/ Tertiary boundary sediments at Kawaruppu, Hokkaido, Japan. Geochem. J. 33, 285-294.

Mita, H., Shimoyama, A. and Kajiwara, Y. (1996) Search for extraterrestrial amino acids in sediments at the Cretaceous/Tertiary boundary in Kawaruppu, Hokkaido, Japan. Geochem. J. 30, 89-98.

Mita, H., Fukunaga, N. and Shimoyama, A. (1998) Characterization of dicarboxylic acids in the Cretaceous/Tertiary boundary sediments at Kawaruppu, Hokkaido, Japan, and comparison with those of carbonaceous chondrites. Geochim. Cosmochim. Acta 62, 3695-3702.

Nomoto, S., Kozono, M., Mita, H. and Shimoyama, A. 
(2001) Structural elucidation of an oxidation product of sedimentary porphyrins by one-pot synthesis of 3-methylphthalimide. Bull. Chem. Soc. Jpn. (in press).

Ocampo, R., Callot, H. J., Albrecht, P. and Kintzinger, J. P. (1984) A novel chlorophyll $c$ related petroporphyrin in oil shale. Tetrahedron Letters $\mathbf{2 5}$, 2589-2592.

Ocampo, R., Bauder, C., Callot, H. J. and Albrecht, P. (1992) Porphyrins from Messel oil shale (Eocene, Germany): Structure elucidation, geochemical and biological significance, and distribution as a function of depth. Geochim. Cosmochim. Acta 56, 745761.

Officer, C. B. and Drake, C. L. (1983) The CretaceousTertiary transition. Science 219, 1383-1390.

Saito, T., Yamanoi, T. and Kaiho, K. (1986) End-Cre- taceous devastation of terrestrial flora in the boreal Far East. Nature 323, 253-255.

Smit, J. and Hertogen, J. (1980) An extraterrestrial event at the Cretaceous-Tertiary boundary. Nature 285, 198200.

Sundararaman, P. and Boreham, C. J. (1990) Vanadyl 3-nor $\mathrm{C}_{30} \mathrm{DPEP}$ : Indicator of depositional environment of a lacustrine sediment. Geochim. Cosmochim. Acta 55, 389-395.

Treibs, A. (1934) The occurrence of chlorophyll derivatives in organic materials. Annalen 517, 103-114.

Verne-Mismer, J., Ocampo, R., Callot, H. J. and Albrecht, P. (1988) Molecular fossils of chlorophyll $c$ of the 17-nor-DPEP series. Structure determination, synthesis, geochemical significance. Tetrahedron Letters 29, 371-374.

\section{APPENDIX}

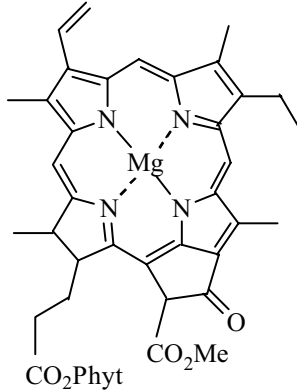

Chlorophyll-a

(Phyt: phytyl)

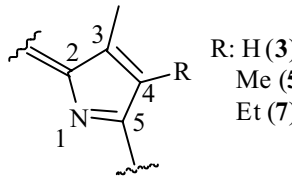

3-Methyl-4-alkylpyrrole unit

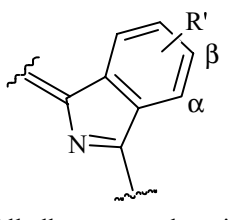

Alkylbenzopyrrole unit

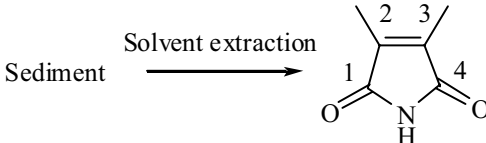

Free 2,3-dimethylmaleimide (1)<smiles>CCC1=C(C)C(=O)NC1=O</smiles>

Free 2-methyl-3-ethylmaleimide (2)

Oxidation [O]

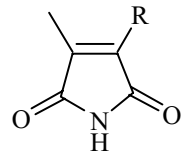

R: $H(4)$

$\operatorname{Me}(6)$

Et (8)

Bound 3-methyl-4-alkylmaleimide
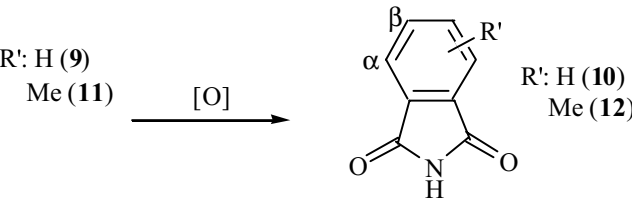

Bound alkylbenzomaleimide 